\title{
Deformable Mirror configuration in MCAO: is propagation a fundamental limit on visible wavelength correction?
}

\author{
Ollie Farley ${ }^{l}$, James Osborn ${ }^{l}$, Tim Morris ${ }^{1}$, Andrew Reeves ${ }^{1}$ and Richard W. Wilson ${ }^{1}$ \\ ${ }^{1}$ Centre for Advanced Instrumentation (CfAI), Durham University, UK
}

\begin{abstract}
To achieve the best correction using a multi-conjugate adaptive optics (MCAO) system, the sequence of the deformable mirrors must be taken into account. Since atmospheric turbulence is sampled from high to low altitude, the subsequent phase corrections must be performed in a symmetrical way (low to high altitude) to avoid aberration arising from optical propagation through the system. This is a well known problem in solar MCAO however ordering the DMs in this way requires bulky relay optics, therefore most post focal MCAO systems for night-time astronomy have been designed with the DMs ordered from high to low altitude. We show that this results in a small wavefront error that can become nonnegligible in the visible. Employing an adaptive secondary mirror conjugated to the ground has the potential to alleviate this effect.
\end{abstract}

\section{INTRODUCTION}

In multi-conjugate adaptive optics (MCAO), multiple phase correcting elements are conjugated to the altitude of turbulent layers in the atmosphere. This can result in an additional source of error due to propagation if the correcting elements are placed in the optical path in the order of highest to lowest altitude. ${ }^{1}$ This is the most common configuration in current and planned MCAO systems ${ }^{2-5}$ since it is more compact and optically efficient, avoiding the need for long optical relays (see Figure 1). The effect is too small to be seen in the infrared wavelengths at which most current MCAO systems operate, but with proposals for visible wavelength $\mathrm{MCAO}^{4,6}$ the residual error from propagation becomes a larger component of the total error budget. This effect and its dependence on turbulence profile and deformable mirror configuration is investigated in simulation, in particular the reduction in wavefront error when employing an adaptive secondary mirror.

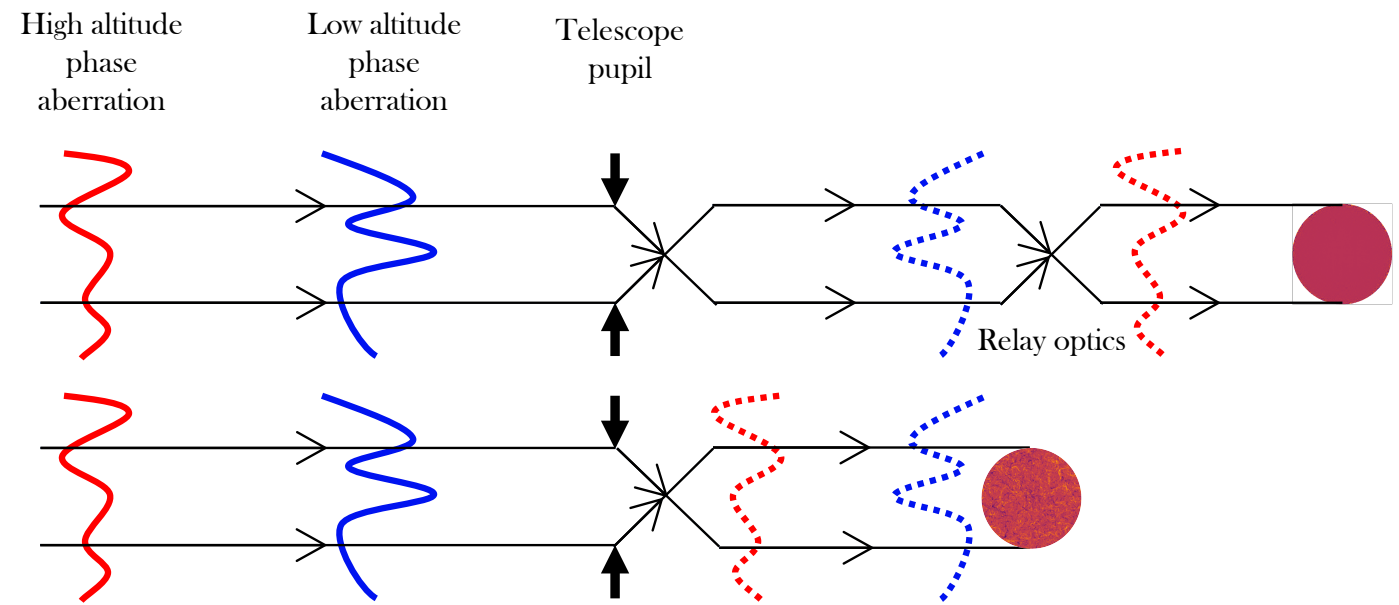

Figure 1. Schematic diagram illustrating the two possible configurations of a post focal two DM MCAO system. Solid red and blue lines represent phase aberration and dotted lines their corresponding correction by DMs. Top: Configuration A, where the low altitude correction is performed first. This results in perfect correction at the expense of a longer optical system requiring relays. Bottom: Configuration B, where the high altitude correction is performed first. This is more compact and optically efficient however results in aberrations from propagation. 

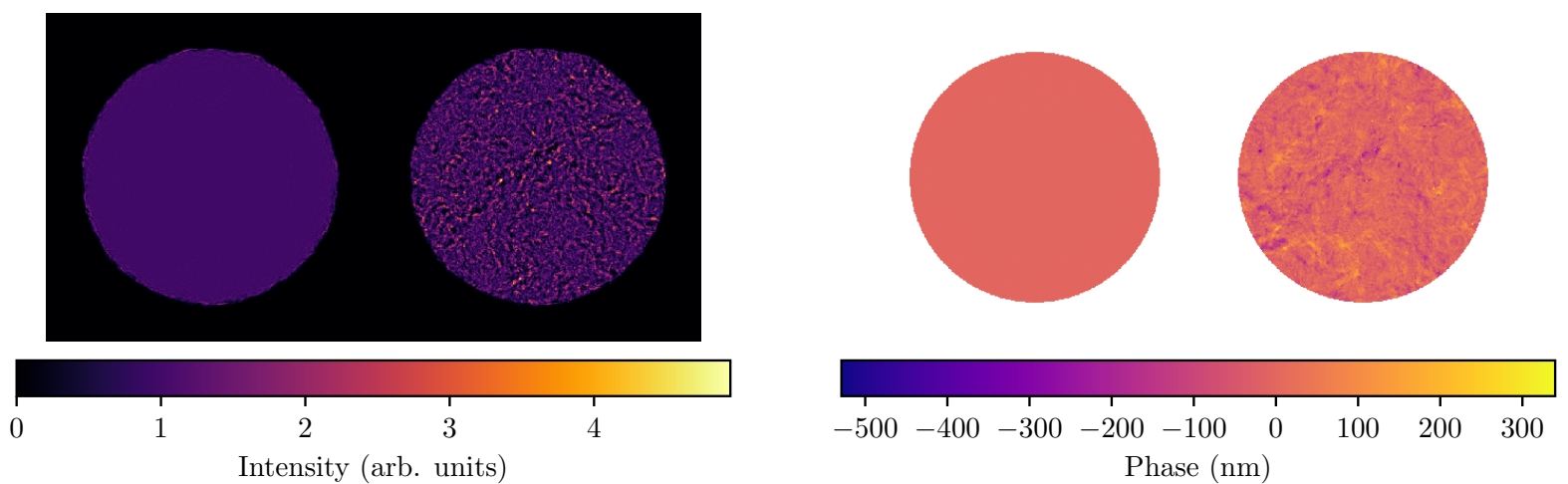

Figure 2. Intensity and phase patterns of the electric fields $E_{\mathrm{A}}$ (left) and $E_{\mathrm{B}}$ (right). Configuration A results in a perfect wavefront with no aberration. Configuration B results in both amplitude (intensity) and phase aberration arising from propagation. Calculated using wave-optical propagation of Monte-Carlo Von Karman phase screens with $r_{0}=16 \mathrm{~cm}$ and a $4 \mathrm{~m}$ aperture.

\section{WAVE-OPTICAL SIMULATION AND RESULTS}

The importance of the order of correcting elements in MCAO for astronomy was investigated by Flicker (2001). ${ }^{1}$ It was found that configuring the correcting elements "backwards" in an optical sense - that is from high altitude to low altitude in the optical path - could affect the Strehl ratio by as much as $10 \%$ at visible wavelengths. Configuring the correcting elements the other way round such that they are symmetrical about the telescope pupil resulted in zero error. The effect can be understood by considering two deformable mirrors, one conjugated to the ground and the other to a high altitude layer at $10 \mathrm{~km}$.

We model wave-optical propagation of the electric field $E$ by the convolution

$$
E\left(x_{2}, y_{2}\right)=E\left(x_{1}, y_{1}\right) \otimes \frac{e^{i k \Delta z}}{i \lambda \Delta z} e^{\frac{i k}{2 \Delta z}\left(x_{1}^{2}+y_{1}^{2}\right)}=E\left(x_{1}, y_{1}\right) \otimes K(\Delta z),
$$

where $x_{1}, y_{1}$ and $x_{2}, y_{2}$ are position coordinates in two planes separated by $\Delta z, k$ the wavevector and $\lambda$ the wavelength of light. The function $K(\Delta z)$ is known as the Fresnel kernel and $\otimes$ denotes the convolution operator. ${ }^{7}$

We define configuration A as the case when the ground correction is performed first, followed by the high altitude correction. Using Equation 1 we can calculate the electric field at the telescope pupil:

$$
E_{\mathrm{A}}=\left[\left[\left[e^{i \phi_{10}} \otimes K(10 \mathrm{~km})\right] e^{i \phi_{0}} P(r) e^{i \phi_{0}^{\prime}}\right] \otimes K(-10 \mathrm{~km}) e^{i \phi_{10}^{\prime}}\right] \otimes K(10 \mathrm{~km}),
$$

where $\phi_{10}$ and $\phi_{0}$ represent phase aberrations due to the atmosphere at $10 \mathrm{~km}$ and the ground respectively, $\phi_{10}^{\prime}$ and $\phi_{0}^{\prime}$ the correction phase provided by the DMs conjugated to their respective altitudes and $P(r)$ is the aperture function representing the telescope aperture, equal to 1 inside the aperture and 0 elsewhere. It can be seen that if the DMs provide perfect correction of the phase aberration, i.e. $\phi_{10}=-\phi_{10}^{\prime}$ and $\phi_{0}=-\phi_{0}^{\prime}$ then this expression cancels to unity; a perfect flat wavefront with no phase or intensity aberration.

In the second configuration, B, the high altitude correction is performed first. The expression for the electric field at the pupil after correction is

$$
E_{\mathrm{B}}=\left[\left[\left[e^{i \phi_{10}} \otimes K(10 \mathrm{~km})\right] e^{i \phi_{0}} P(r)\right] \otimes K(-10 \mathrm{~km}) e^{i \phi_{10}^{\prime}}\right] \otimes K(10 \mathrm{~km}) e^{i \phi_{0}^{\prime}} .
$$

The perfect correction case does not cancel to unity here as the combination of ground layer and high altitude phase aberration are propagated together before any correction. During this propagation the complex amplitude and phase from the two layers mix and the corrections $\phi_{0}^{\prime}$ and $\phi_{10}^{\prime}$ are no longer accurate, hence after correction an error is induced. Here we will focus on the phase (wavefront) error since it contributes more to image quality, however both intensity and phase errors arise from this effect, as can be seen in Figure 2.

In order to quantify the level of this induced wavefront error (WFE) a simple wave-optical simulation of an MCAO system with two DMs was used. Atmospheric turbulence was simulated using random phase screens with a Von Karman 

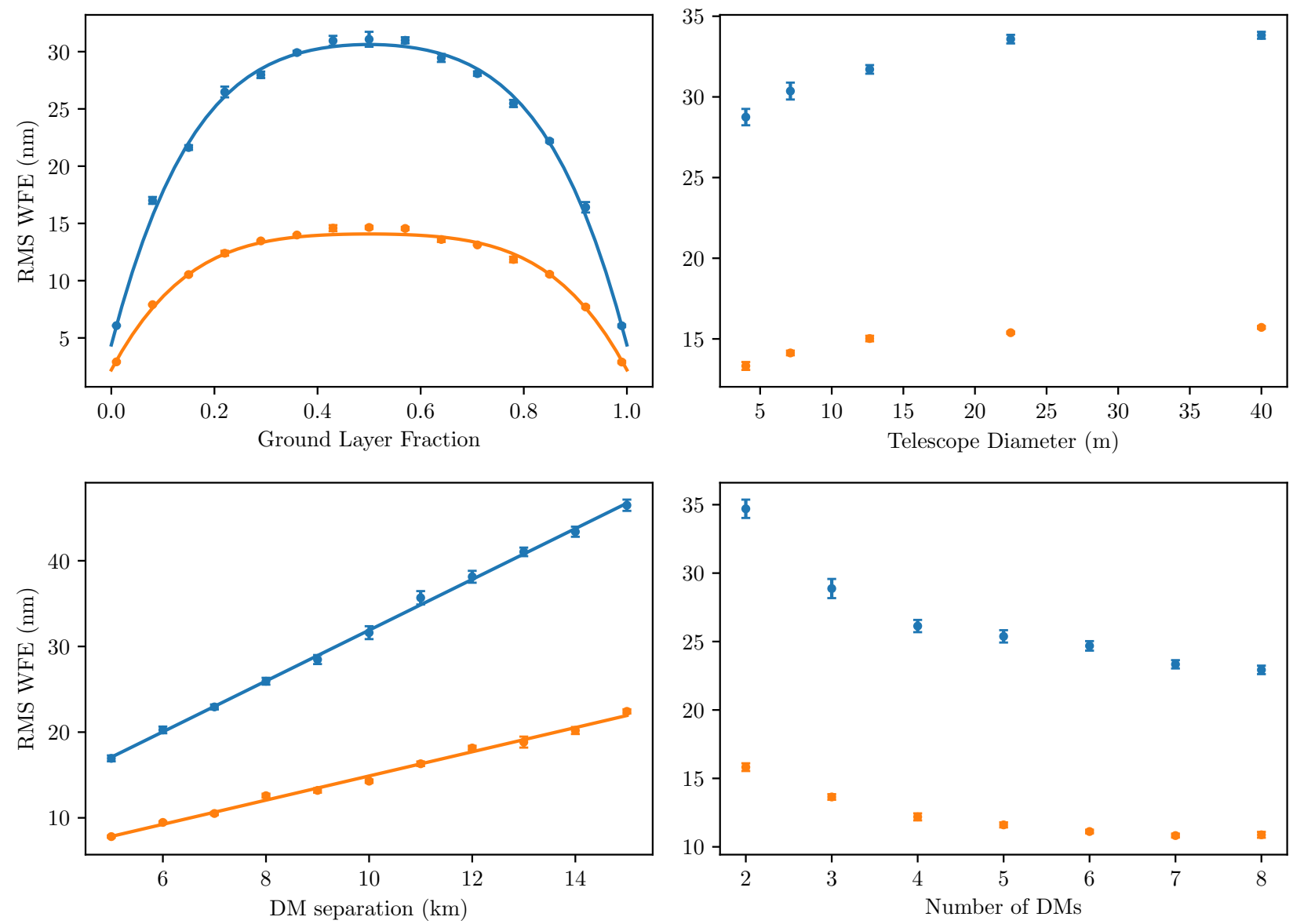

Figure 3. Dependencies of the induced onaxis RMS WFE after MCAO correction in configuration B. Blue data is for $r_{0}=10 \mathrm{~cm}$ and orange data is for $r_{0}=16 \mathrm{~cm}$. Default parameters unless stated otherwise: $D=8 \mathrm{~m}, 2$ DMs conjugated to turbulent layers at the ground and $10 \mathrm{~km}$, ground layer fraction 0.5 . DMs are always perfectly conjugated to a turbulent layer. $\lambda=500 \mathrm{~nm}$. Top Left: WFE dependence on ground layer fraction, i.e. how much turbulence is corrected by each DM. The fitted curves are fourth-order polynomials. Top Right: WFE dependence on telescope diameter. Bottom Left: WFE dependence on DM separation, with linear fit. Bottom Right: WFE dependence on the number of DMs $n$ in the turbulent volume between 0 and $10 \mathrm{~km}$. Note that the turbulence in this case is spread between $n$ turbulent layers.

power spectrum generated using the AOTools library. ${ }^{8}$ Propagation was performed using the angular spectrum algorithm. ${ }^{7,8}$ The phase correction of the DMs is simulated by simply subtracting the relevant phase screen, thus we are emulating a perfect DM. This allows us to focus on the error induced by propagation. The post-MCAO electric fields are calculated for each configuration A and B using Equations 2 and 3 respectively. The phase of these electric fields is then recovered using a phase unwrapping algorithm. ${ }^{9}$

The results of these simulations are shown in Figure 3. We find that the greatest phase aberration occurs when the turbulence is evenly distributed between two layers. The magnitude of the error is proportional to the magnitude of the input aberration, i.e. the integrated turbulence strength. For $r_{0}=10 \mathrm{~cm}$ we obtain approximately $30 \mathrm{~nm}$ phase aberration and for $r_{0}=16 \mathrm{~cm}$ we obtain around $13 \mathrm{~nm}$ phase aberration. The peak of the distribution is reasonably flat therefore for stronger ground layers (up to around $75 \%$ ground layer fraction) there is not a large change from the peak value. The variation with telescope diameter is fairly weak, with the WFE increasing by less than $20 \%$ from $29 \mathrm{~nm}$ to only 34 nm between $D=4 \mathrm{~m}$ and $D=40 \mathrm{~m}$, an order of magnitude in aperture diameter. It should be noted that we have not considered turbulence the outer scale $L_{0}$ here, i.e. $L_{0} \gg D$ for all values of $D$. The dependence on DM separation distance is linear in the chosen range, with the gradient proportional again to the integrated turbulence strength. This is expected, as a greater separation between DMs means greater propagation distance and hence greater error. Finally the decrease in 


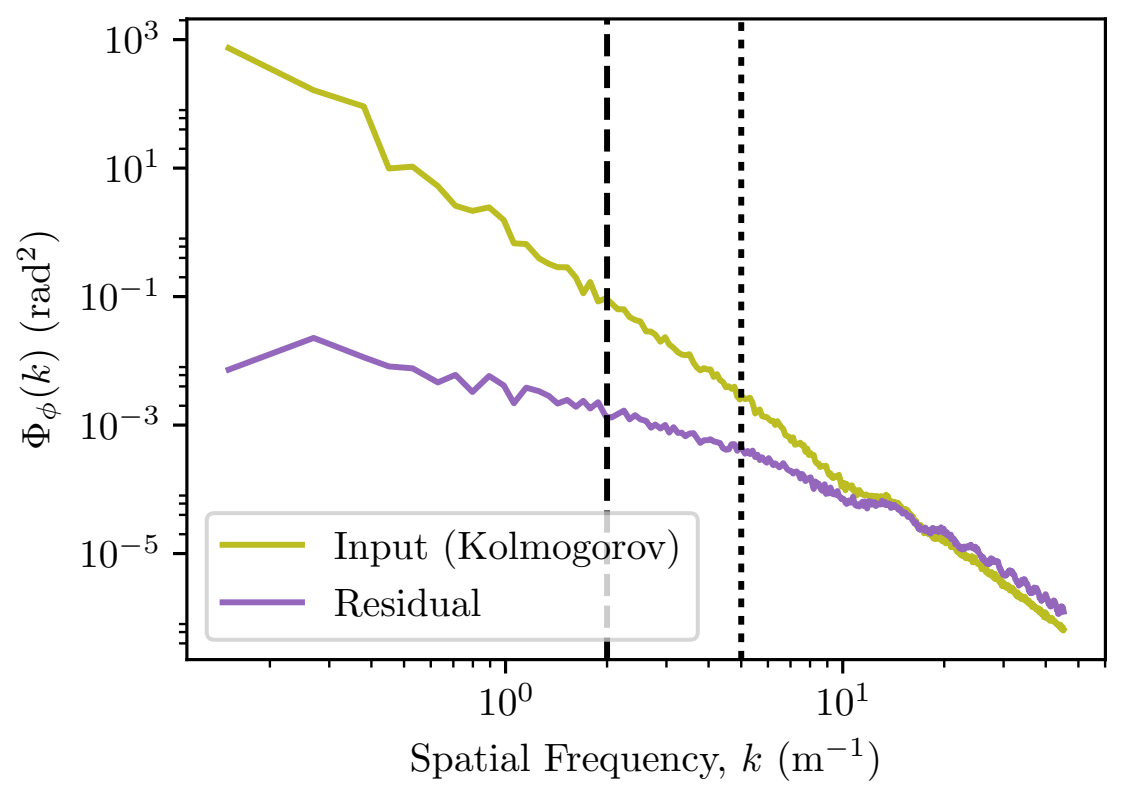

Figure 4. Spatial power spectral density from Monte Carlo wave-optical simulation of phase across an $8 \mathrm{~m}$ aperture both pre- (input) and post-MCAO correction (residual). The DMs are separated by $10 \mathrm{~km}$, with the atmosphere having $r_{0}=10 \mathrm{~cm}$ split evenly between a layer at $10 \mathrm{~km}$ and a layer at the ground. The dashed and dotted vertical lines represent spatial frequency cutoffs for subaperture sizes of $50 \mathrm{~cm}$ and $20 \mathrm{~cm}$ respectively.

WFE with the number of DMs is shown. An increase in the number of DMs in the same turbulent volume acts to decrease the propagation distances thereby reducing the overall WFE.

It is not only the magnitude of overall WFE but the spatial structure of the phase aberration that is of interest. The spatial power spectrum is shown in Figure 4. Here we can see that the pre-MCAO input has the expected Kolmogorov $-11 / 3$ slope with some modulation due to atmospheric scintillation. Post-MCAO the residual phase aberration has a much flatter profile, with low spatial frequencies being fairly effectively suppressed but high frequency variation being the same, even slightly amplified compared to the input aberration. The interest here lies in the fact that all MCAO systems will operate in closed loop, thus the wavefront sensors will "see" this additional phase aberration resulting from propagation through the DMs. The simplest approach to modelling this would be to treat the AO system as a high-pass spatial filter, removing the low-order aberrations (those to the left of the dotted and dashed lines in Figure 4). This would result in a reduction in WFE, however the power spectrum shows that there is still significant power in the high spatial frequencies. This simplistic assumption may not bear weight however since tomographic reconstructors are complex non-linear systems ${ }^{10}$ and the effect of this propagation induced WFE with these reconstructors is unclear. A full end-to-end MCAO simulation with physical propagation would be required to properly assess this effect.

There is an interesting middle ground between configurations A and B: that of a pre-focal deformable secondary mirror conjugated to the ground that performs the ground layer correction first before any post-focal high altitude DMs. Since most of the turbulence is usually in the ground layer correcting this phase aberration in the "correct" order reduces the induced WFE considerably even if there are multiple post-focal DMs configured as in configuration B. Adaptive secondary mirrors form the basis of the MCAO designs for MAORY on the E-ELT, ${ }^{5}$ the proposed visible wavelength upgrade to the VLT AOF ${ }^{4}$ and the GMT MCAO system ${ }^{11}$ (although it should be noted that the DMs of the GMT MCAO system use configuration A therefore this effect is irrelevant for that telescope).

A summary of the expected impact on Strehl ratio for real and planned MCAO systems is shown in Figure 5. These simulations used a simple model atmosphere with $65 \%$ of the turbulence in the ground layer, the remaining $35 \%$ being split between the altitude conjugated DMs. It can be seen that in infrared wavelengths the effect of physical propagation is small but as we move towards the visible the effect can become non-negligable. The most striking example here is NFIRAOS, the MCAO system for the Thirty Meter Telescope (TMT), which suffers around a 15\% reduction in Strehl ratio 


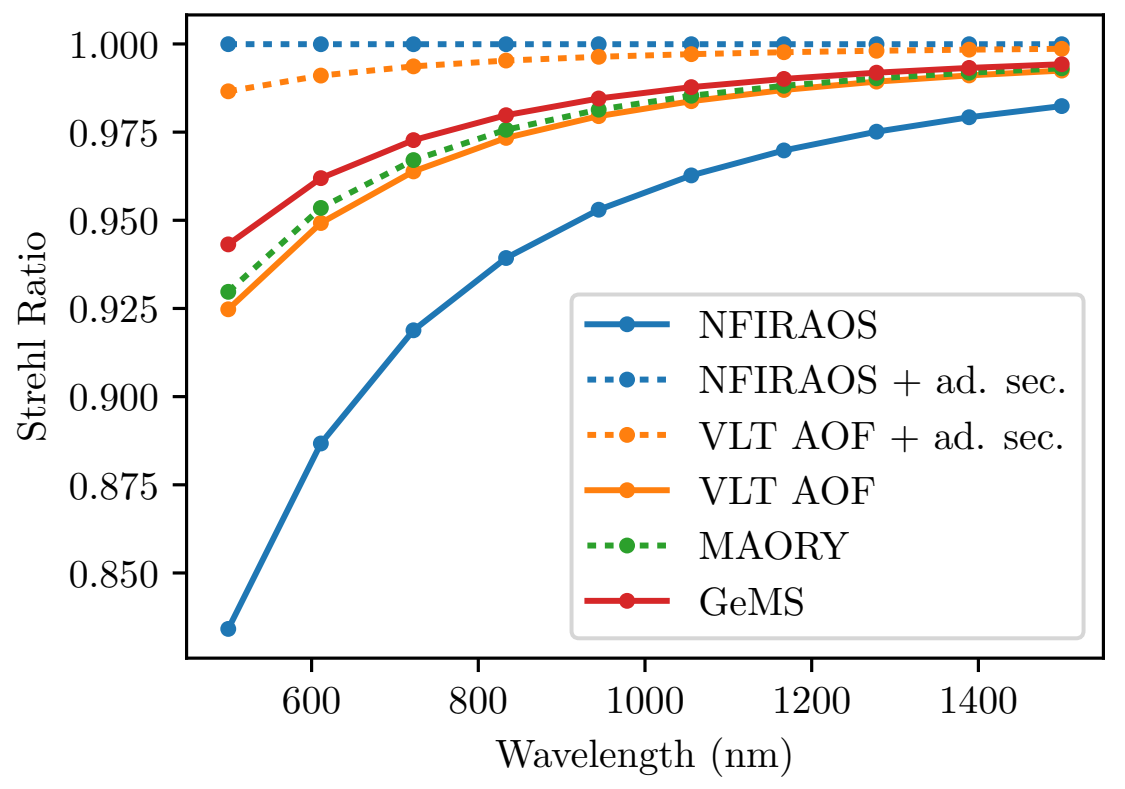

Figure 5. Impact of physical propagation on the onaxis Strehl ratio of real and planned MCAO systems. Dotted lines represent systems with pre-focal adaptive secondary mirrors. The simplified MCAO system used for simulation provides perfect correction in all other ways, therefore any loss in Strehl ratio arises only from the propagation effect and will add to other errors (DM fitting, anisoplanatism, etc).

at $500 \mathrm{~nm}$. This is due to the long conjugate distance between the two DMs (around $11 \mathrm{~km}$ ). By replacing the post-focal ground conjuated DM with a pre-focal deformable secondary, shown in the dashed blue line, we completely negate this effect. Systems with $3 \mathrm{DMs}$ generally perform better due to reduced propagation distances with further reduction when considering an adaptive secondary mirror. Another notable case is MAORY, the proposed E-ELT MCAO system. This suffers an $8 \%$ loss in Strehl, much worse than the other systems emplying adaptive secondaries. This is again due to long propagation distances: the post-focal DMs are conjugated to $15.5 \mathrm{~km}$ and $4.5 \mathrm{~km}$ in the current plans. ${ }^{12}$ All simulations have been perfomed at zenith but it has been shown that airmass is also a factor in further reducing the Strehl ratio due to this effect.

\section{CONCLUSIONS}

It has been shown empirically using wave-optical simulation of a simplified MCAO system that the propagation induced WFE was proportional to the integrated turbulence strength as well as the propagation distance between the two DMs when configured from high to low conjugate altitudes in the optical path. The WFE was found to depend weakly on both the telescope diameter and the distribution of turbulence between the two DMs. The magnitude of the WFE in strong turbulence and in MCAO systems with long conjugate distances between DMs is enough to become a small but non-negligable part of the error budget in the visible, of the order of tens of nm RMS. This has the potential to reduce the achievable Strehl ratio by as much as $15 \%$ on NFIRAOS at $500 \mathrm{~nm}$. Although most systems such as NFIRAOS are designed to operate in the infrared, this work shows it is desirable to employ a pre-focal adaptive secondary mirror if pushing into visible wavelength MCAO. Further simulations with a full end-to-end MCAO model are required to ascertain the ability of a closed loop wavefront sensor and tomographic reconstructor to sense and correct this WFE.

\section{Acknowledgements}

This work is supported by STFC (ST/N50404X/1). 


\section{REFERENCES}

[1] Flicker, R. C., "Sequence of phase correction in multiconjugate adaptive optics," Optics Letters 26, 1743 (Nov 2001 ).

[2] Bec, M., Rigaut, F. J., Galvez, R., Arriagada, G., Boccas, M., Gausachs, G., Gratadour, D., James, E., Rojas, R., Rogers, R., Sheehan, M. P., Trancho, G., and Vucina, T., "The Gemini MCAO bench: system overview and lab integration," in [Proceedings of SPIE], Hubin, N., Max, C. E., and Wizinowich, P. L., eds., 7015, 701568 (Jul 2008 ).

[3] Herriot, G., Andersen, D., Atwood, J., Boyer, C., Byrnes, P., Caputa, K., Ellerbroek, B., Gilles, L., Hill, A., Ljusic, Z., Pazder, J., Rosensteiner, M., Smith, M., Spano, P., Szeto, K., Véran, J.-P., Wevers, I., Wang, L., and Wooff, R., "NFIRAOS: first facility AO system for the Thirty Meter Telescope," in [Proceedings of SPIE], Marchetti, E., Close, L. M., and Véran, J.-P., eds., 9148, 914810 (Jul 2014).

[4] Esposito, S., Agapito, G., Bonaglia, M., Busoni, L., Fusco, T., Neichel, B., Spano, P., Bono, G., and Vernet, J., "AOF upgrade for VLT UT4: an $8 \mathrm{~m}$ class HST from ground," in [Proceedings of SPIE], Marchetti, E., Close, L. M., and Véran, J.-P., eds., 9909 (Jul 2016).

[5] Lombini, M., De Rosa, A., Ciliegi, P., Cortecchia, F., Diolaiti, E., Patti, M., Bonaglia, M., Busoni, L., De Caprio, V., Esposito, S., Feautrier, P., Rabou, P., Riva, M., and Stadler, E., "Optical design of the post-focal relay of MAORY," in [Proceedings of SPIE], Evans, C. J., Simard, L., and Takami, H., eds., 9908, 9908AB (Aug 2016).

[6] Ragazzoni, R., Herbst, T., Gaessler, W., Andersen, D., Arcidiacono, C., Baruffolo, A., Baumeister, H., Bizenberger, P., Diolaiti, E., Esposito, S., Farinato, J., Rix, H., Rohloff, R.-R., Riccardi, A., Salinari, P., Soci, R., Vernet-Viard, E., and Xu, W., "A visible MCAO channel for NIRVANA at the LBT," in [Proceedings of SPIE], 4839 (2002).

[7] Schmidt, J. D., [Numerical Simulation of Optical Wave Propagation with Examples in MATLAB], SPIE (2010).

[8] "AOTools: A useful set of tools for Adaptive Optics in Python." https://github.com/AOtools/aotools. Accessed: 2017-08-10.

[9] van der Walt, S., Schönberger, J. L., Nunez-Iglesias, J., Boulogne, F., Warner, J. D., Yager, N., Gouillart, E., Yu, T., and the scikit-image contributors, "scikit-image: image processing in Python," PeerJ 2, e453 (Jun 2014).

[10] Fusco, T., Conan, J.-m., Rousset, G., Mugnier, L. M., and Michau, V., "Optimal wave-front reconstruction strategies for multiconjugate adaptive optics," Journal of the Optical Society of America A 18, 2527 (Oct 2001).

[11] Hinz, P. M., Bouchez, A., Johns, M., Shectman, S., Hart, M., McLeod, B., and McGregor, P., "The GMT adaptive optics system," in [Proceedings of SPIE], Ellerbroek, B. L., Hart, M., Hubin, N., and Wizinowich, P. L., eds., 7736, 77360C (Jul 2010).

[12] Diolaiti, E. et al., "On the road to the Preliminary Design Review of the MAORY adaptive optics module for E-ELT," in [AO4ELT5], (Jul 2017). 